PROCEEDINGS OF THE

AMERICAN MATHEMATICAL SOCIETY

Volume 124, Number 10, October 1996

\title{
SEPARATING VERSUS STRICTLY SEPARATING VECTORS
}

\author{
EDWARD A. AZOFF, LIFENG DING, AND WARREN R. WOGEN \\ (Communicated by Palle E. T. Jorgensen)
}

\begin{abstract}
Let $S$ be a linear manifold of Banach space operators which is closed in the strong operator topology. Existence of a disjoint pair of separating vectors does not guarantee reflexivity of $S$, but $S$ must be reflexive if one of these vectors is strictly separating. $S$ must also be reflexive if all non-zero linear combinations of some independent pair of vectors strictly separate $S$.
\end{abstract}

\section{INTRODUCTION}

Throughout this paper, $X$ and $Y$ denote complex Banach spaces, and we write $B(X, Y)$ for the collection of all bounded linear operators acting between them.

Definition 1.1. Let $S$ be a linear subspace of $B(X, Y)$.

(1) The reflexive closure of $S$ is defined by the equation

ref $S=\{b \in B(X, Y): b x$ belongs to the norm closure of $S x$ for each $x \in X\}$.

(2) $S$ is said to be reflexive if $S=\operatorname{ref} S$.

Thus ref $S$ consists of all operators which belong to $S$ "locally". When $X=Y$ and $S$ is an identity-containing operator algebra, ref $S$ coincides with alg lat $S$. The more general point of view of Definition 1.1 is due to A. I. Loginov and V. S. Shulman. This additional flexibility has proved quite useful in constructing counterexamples; there are various techniques for embedding pathological operator spaces in pathological operator algebras [1], [2], [3], [7].

Definition 1.2. Let $S$ be a linear subspace of $B(X, Y)$ and suppose $x \in X$.

(1) The evaluation map $f_{x}: S \rightarrow Y$ is defined by

$$
f_{x}(b)=b x .
$$

(2) The vector $x \in X$ separates $S$ if $f_{x}$ is injective on $S$.

(3) The vector $x \in X$ strictly separates $S$ if $f_{x}$ is bounded below on $S$.

There are several results in the literature concerning the relationship between reflexivity and strictly separating vectors. The present paper shows that the assumption of strict separation is more than a matter of convenience. Before stating our main results, we review the standard counterexample concerning reflexivity and separation.

Received by the editors November 28, 1994 and, in revised form, April 3, 1995.

1991 Mathematics Subject Classification. Primary 47D15.

Key words and phrases. Reflexive operator space, separating vector, strictly separating vector.

(C)1996 American Mathematical Society 
Example 1.3. Take $X=Y=\mathbb{C}^{2}$ and set

$$
S=\left\{\left[\begin{array}{ll}
\alpha & \beta \\
0 & \alpha
\end{array}\right]: \alpha, \beta \in \mathbb{C}\right\}
$$

On the one hand, $S$ has many separating vectors - the only vectors which fail to separate $S$ are scalar multiples of $(1,0)$. By finite-dimensionality, all separating vectors are automatically strictly separating.

On the other hand, it is easy to check that $S$ fails to be reflexive- every uppertriangular matrix belongs to ref $S$.

The "disjointness" hypotheses on the separating vectors in the following two theorems should be thought of as minimal ways to avoid the pathology of Example 1.3. These are the main positive results of the paper.

Theorem 1.4. Let $S$ be a subspace of $B(X, Y)$ which is closed in the strong operator topology. Suppose $S$ admits a separating vector $x$ and a strictly separating vector y satisfying $S x \cap S y=\{0\}$. Then $S$ is reflexive.

Theorem 1.5. Let $S$ be a subspace of $B(X, Y)$ which is closed in the strong operator topology. If there is a two-dimensional subspace of $X$ whose non-zero members all strictly separate $S$, then $S$ is reflexive.

In Section 2, we establish the analyticity of certain operator-valued maps associated with strictly separating vectors. This is crucial to the proofs of Theorems 1.4 and 1.5, presented in Section 3. Theorems 1.4 and 1.5 are independent generalizations of the main result of [5]; examples to this effect are also given in Section 3.

In Section 4, we show that dropping "strictly" from the hypothesis of Theorem 1.4 destroys its veracity. The relevant counterexample has several additional features, summarized in the following theorem.

Theorem 1.6. There is a strongly closed algebra A of Hilbert space operators with the following properties:

(1) A is singly generated.

(2) A admits separating vectors $x$ and $y$ such that $A x \perp A y$.

(3) $A$ is not reflexive.

The paper closes by reviewing an example of A. Lambert [6, page 724]which rules out weakening of the hypothesis of Theorem 1.5.

Theorem 1.7. There is a strongly closed algebra A of Hilbert space operators with the following properties:

(1) A is singly generated.

(2) Every non-zero vector separates A; some of these vectors are strictly separating.

(3) $A$ is not reflexive.

The reader is referred to [4] for analogues of Theorems 1.4 and 1.5 in the setting of algebraic reflexivity; it is necessary to assume finite dimensionality of $S$ in the algebraic analogue of 1.5 . 


\section{MAPS ASSOCIATED WITH STRICTLY SEPARATING VECTORS}

Throughout this section, $S$ will denote a norm-closed linear subspace of $B(X, Y)$ and we write $U$ for its collection of strictly separating vectors.

Proposition 2.1. In order for a separating vector $x$ to strictly separate $S$ it is necessary and sufficient that $S x$ be a closed subspace of $Y$.

Proof. Apply the Open Mapping Theorem.

Proposition 2.2. Suppose $S$ admits a strictly separating vector. Then $S$ is closed in the strong operator topology and the relative norm and strong operator topologies agree on $S$.

Proof. Suppose $\left\{s_{n}\right\}$ is a net in $S$ converging strongly to an operator $b \in B(X, Y)$. Writing $x$ for some strictly separating vector for $S$, we note that $\left\{s_{n} x\right\}$ is a Cauchy net in $Y$, whence the net $\left\{s_{n}\right\}$ is norm-Cauchy and hence converges to $b$ in norm.

Proposition 2.3. Let $S$ be a linear subspace of $B(X, Y)$.

(1) The collection $U$ of strictly separating vectors for $S$ is an open subset of $X$.

(2) Given a compact subset $K$ of $U$, there is an $\epsilon>0$ such that $\|s x\| \geq \epsilon\|s\|$ for all $s \in S$ and $x \in K$.

Proof. For (1), let $s \in S$ and $x, y \in X$. By the triangle inequality, we have

$$
\left\|f_{y}(a)\right\| \geq\left\|f_{x}(a)\right\|-\left\|f_{x-y}(a)\right\| \geq\left\|f_{x}(a)\right\|-\|x-y\|\|a\| .
$$

Thus $f_{x}$ bounded below implies $f_{y}$ bounded below for $y$ sufficiently close to $x$.

If the conclusion of (2) failed, there would be sequences $\left\{s_{n}\right\} \in S$ and $\left\{x_{n}\right\} \in K$ with $\left\|s_{n}\right\|=1$ for all $n$ and $\lim s_{n} x_{n}=0$. Dropping down to a subsequence if necessary, we may assume the $\left\{x_{n}\right\}$ converge to some $y \in K$, whence $\lim s_{n} y=$ 0 by the triangle inequality. That, however would contradict $y \in U$, so (2) is established.

Definition 2.4. Given $b \in \operatorname{ref} S$ and $x \in U$, we write $g_{b}(x)$ for the unique operator in $S$ satisfying $g_{b}(x) x=b x$.

Thus $g_{b}$ is an operator-valued function defined on the collection of strictly separating vectors. The next proposition explains its relevance to the proofs of Theorems 1.4 and 1.5 .

Proposition 2.5. Let $S$ be a norm-closed linear subspace of $B(X, Y)$ admitting a strictly separating vector $x$. Then the following are equivalent.

(1) $S$ is reflexive.

(2) For each $b \in$ ref $S$, the function $g_{b}$ is constant in a neighborhood of $x$.

Proof. It is clear that (1) implies (2) since $g_{b}$ is constant when $b \in S$.

For the converse, assume (2) and suppose $b \in \operatorname{ref} S$. Set $s=g_{b}(x)$. By hypothesis, we have $s y=b y$ for all $y$ in some neighborhood of $x$ whence $b=s \in S$ by linearity.

Proposition 2.6. For each $b \in$ ref $S$ the function $g_{b}$ is continuous on its domain. 
Proof. Fix $b$ and suppose $\left\{x_{n}\right\}$ is a sequence in $U$ converging to $x_{0} \in U$. For each $n$, set $s_{n}=g_{b}\left(x_{n}\right)$. It is a matter of definition and continuity of $b$ that

$$
\lim s_{n} x_{n}=\lim b x_{n}=b x_{0}=s_{0} x_{0} .
$$

But by Proposition 2.3.2, we know that the $\left\{s_{n}\right\}$ are uniformly bounded whence $\lim s_{n} x_{0}=s_{0} x_{0}$ as well. Because $x_{0}$ strictly separates $S$, we get $\lim s_{n}=s_{0}$ as desired.

Definition 2.7. Suppose $b \in \operatorname{ref} S, x$ strictly separates $S$, and $y$ is any vector in $X$. Write $V$ for the set of complex numbers $\lambda$ such that $x+\lambda y \in U$ and define the operator-valued function $h_{x, y}: V \rightarrow S$ by setting $h_{x, y}(\lambda)=g_{b}(x+\lambda y)$.

In other words, $h_{x, y}(\lambda)$ is the unique member of $S$ satisfying

$$
h_{x, y}(\lambda)(x+\lambda y)=b(x+\lambda y) .
$$

For simplicity, the notation suppresses dependence of $h$ on $b$; when there is no danger of confusion reference to $x, y$ will also be omitted. It follows from Proposition 2.6 that $h_{x, y}$ is continuous throughout its domain.

Proposition 2.8. The function $h_{x, y}$ is analytic throughout its domain and $h_{x, y}^{\prime}(0) x$ $=\left(b-h_{x, y}(0)\right) y$.

Proof. Suppose $\lambda, \lambda_{0} \in V$. Manipulation of Equation (2-1) yields

$$
\frac{h(\lambda)-h\left(\lambda_{0}\right)}{\lambda-\lambda_{0}}\left(x+\lambda_{0} y\right)=b y-h(\lambda) y .
$$

Applying the continuity of $h$, we conclude that

$$
\lim _{\lambda \rightarrow \lambda_{0}} \frac{h(\lambda)-h\left(\lambda_{0}\right)}{\lambda-\lambda_{0}}\left(x+\lambda_{0} y\right)=b y-h\left(\lambda_{0}\right) y
$$

converges in $Y$. Since $x+\lambda_{0} y$ strictly separates $S$ and all of the difference quotients involved belong to $S\left(x+\lambda_{0} y\right)$, we learn that

$$
\lim _{\lambda \rightarrow \lambda_{0}} \frac{h(\lambda)-h\left(\lambda_{0}\right)}{\lambda-\lambda_{0}}
$$

converges in norm to a member of $S$, thereby establishing analyticity. The proof is completed by substituting $\lambda_{0}=0$ in Equation (2-2).

\section{Positive Results}

In this section, we use Proposition 2.8 to complete the proofs of Theorems 1.4 and 1.5. Relevant corollaries and counterexamples are then discussed.

Proposition 3.1. Suppose $c \in$ ref $S$ satisfies $c x=0$ for some strictly separating vector $x$. Then the range of $c$ is contained in $S x$.

Proof. Fix $z \in X$. The hypothesis $c x=0$ means that $h_{x, z}(0) x=0$. It therefore follows from Proposition 2.8 that $c z=h_{x, z}^{\prime}(0) x$. On the other hand, $h_{x, z}^{\prime}(0) x$ is a limit of difference quotients belonging to $S x$, whence $c z \in S x$ as desired.

Theorem 1.4. Let $S$ be a subspace of $B(X, Y)$ which is closed in the strong operator topology. Suppose $S$ admits a separating vector $x$ and a strictly separating vector y satisfying $S x \cap S y=\{0\}$. Then $S$ is reflexive. 
Proof. Replacing $x$ by $\epsilon x+y$ for sufficiently small $\epsilon$ if necessary, we may as well assume that $x, y$ both strictly separate $S$.

Let $b \in \operatorname{ref} S$. Then $c=b-g_{b}(x)$ still belongs to ref $S$ and satisfies $c x=0$, whence range $c \subset S x$ by Proposition 3.1. In particular, $c y \in S x \cap S y$ so $c y=0$ as well. Thus a second application of Proposition 3.1 yields range $c \subset S x \cap S y$. This means $c=0$, so $b=g_{b}(x) \in S$ as desired.

Lemma 3.2. Suppose $x, y$ are independent vectors in $X$ whose non-zero linear combinations all strictly separate $S$. Then if $z \in X$ is sufficiently close to $y$, it is also true that all non-zero linear combinations of $x, z$ strictly separate $S$.

Proof. Applying a compactness argument to the unit sphere of $\mathbb{C}^{2}$ yields $\epsilon>0$ satisfying

$$
\|s(\lambda x+\mu y)\| \geq 2 \epsilon\|s\| \max (|\lambda|,|\mu|) \quad \text { whenever } s \in S, \text { and } \lambda, \mu \in \mathbb{C} .
$$

Thus if $\|z-y\|<\epsilon$, the triangle inequality yields

$$
\|s(\lambda x+\mu z)\| \geq \epsilon\|s\| \max (|\lambda|,|\mu|) \quad \text { whenever } s \in S, \text { and } \lambda, \mu \in \mathbb{C} .
$$

Theorem 1.5. Let $S$ be a subspace of $B(X, Y)$ which is closed in the strong operator topology. If there is a two-dimensional subspace of $X$ whose non-zero members all strictly separate $S$, then $S$ is reflexive.

Proof. Fix a basis $x, y$ for the two-dimensional subspace mentioned in the theorem and suppose $z$ is sufficiently close to $y$ to satisfy the conclusion of Lemma 3.2. Proposition 2.8 tells us that the function $h_{x, z}$ is entire. Moreover, reference to Equation (2-1) makes it clear that $h_{x, z}(\lambda)=h_{z, x}\left(\frac{1}{\lambda}\right)$ for $\lambda \neq 0$. In particular, $h_{x, z}$ is bounded at $\infty$ and hence constant by Liouville's Theorem. A second appeal to Equation (2-1) yields $b z=b x$. Thus $b$ is constant in a neighborhood of $y$, and the proof is completed by applying Proposition 2.5.

Remark 3.3. Review of the proof of Theorem 1.4 shows that it remains valid for real Banach spaces (replace "analytic" by "differentiable" in Proposition 2.8). The real analogue of Theorem 1.5, on the other hand, fails in the simple case when $S=\mathbb{C}$ acts on $\mathbb{R}^{2}$ by complex multiplication.

Definition 3.4. Two subspaces $M, N$ of a Banach space are strongly disjoint if the distance between their unit spheres is strictly positive.

Corollary 3.5. Let $S$ be a subspace of $B(X, Y)$ which is closed in the strong operator topology. Suppose $S$ admits strictly separating vectors $x, y$ such that $S x$ and Sy are strongly disjoint subspaces of $Y$. Then $S$ is reflexive.

Corollary 3.5 is the main result of [5]. It is clear that the hypothesis of strong disjointness implies that $S x \cap S y=\{0\}$. It also yields a number $\delta>0$ satisfying

$$
\|s(\lambda x+\mu y)\| \geq \delta(|\lambda|\|s x\|+|\mu|\|s y\|) \quad \text { for all } s \in S \text { and } \lambda, \mu \in \mathbb{C} \text {, }
$$

whence every non-zero linear combination of $x, y$ must strictly separate $S$. In other words, Theorems 1.4 and 1.5 each generalize Corollary 3.5. The remaining examples in this section show that 1.4 and 1.5 are independent, strict generalizations of 3.5.

Example 3.6. An operator space satisfying the hypothesis of 1.5 but not satisfying the hypothesis of 1.4 . 
Construction. Take $S \subset B\left(\mathbb{C}^{2}, \mathbb{C}^{3}\right)$ represented matricially by

$$
S=\left\{\left[\begin{array}{ll}
\alpha & 0 \\
\beta & \alpha \\
0 & \beta
\end{array}\right]: \alpha, \beta \in \mathbb{C}\right\} \text {. }
$$

It is easy to check that all non-zero linear combinations of $x=\left[\begin{array}{l}1 \\ 0\end{array}\right]$ and $y=\left[\begin{array}{l}0 \\ 1\end{array}\right]$ strictly separate $S$, even though the non-zero vector $\left[\begin{array}{l}0 \\ 1 \\ 0\end{array}\right] \in S x \cap S y$.

Example 3.7. An operator space satisfying the hyposthesis of 1.4 but not satisfying the hypothesis of 1.5 .

Construction. The hypothesis of 1.4 does force all non-zero linear combinations of $x, y$ to separate $S$, so we must look for an infinite-dimensional example.

Take $X=\mathbb{C}^{2}$ and $Y=L_{2}[0,1] \oplus L_{2}[0,1]$. Write $T$ for the position operator, that is

$$
(T f)(t)=t f(t), \quad t \in[0,1] ; \quad f \in L_{2} .
$$

For each $f \in L_{2}$, define an operator $s_{f} \in B(X, Y)$ by setting $s_{f}\left[\begin{array}{l}\lambda \\ \mu\end{array}\right]=\left[\begin{array}{c}\lambda f \\ \mu T f\end{array}\right]$. The desired example is $S=\left\{s_{f}: f \in L^{2}\right\}$.

The absence of eigenvalues for $T$ means that every non-zero vector separates $S$. In fact, $S\left[\begin{array}{l}1 \\ \mu\end{array}\right]$ is precisely the graph of $\mu T$. These spaces are all closed and each pair of them intersects trivially, so the hypothesis of Theorem 1.4 is indeed satisfied. On the other hand, since the range of $T$ fails to be closed, we see that $S\left[\begin{array}{l}0 \\ 1\end{array}\right]$ is not closed either, thereby violating the hypothesis of Theorem 1.5.

\section{OPERATOR SPACES AND BLOCK MATRICES}

Throughout this section, $H$ will denote a separable, infinite-dimensional Hilbert space.

Example 4.1. There is a closed operator $b$ on $H$, a bounded linear functional $\phi \in H^{*}$ and an injective operator $c \in B(H)$ such that given $\lambda \in \mathbb{C}$ and $\epsilon>0$, one can find a vector $x \in H$ satisfying:

(1) $b x=\lambda x$,

(2) $\phi x=1$, and

(3) $\|c x\|<\epsilon$.

Construction. Write $\left\{f_{n}\right\}$ for the standard orthonormal basis of $\ell_{2}$, and $d$ for the diagonal operator on $\ell_{2}$ with diagonal entries $\left\{\frac{1}{n^{2}}\right\}$. We also let $a$ act on the Hardy space $H^{2}(\mathbb{T})$ by differentiation. It is well-known and easy to verify that $a$ is a closed operator. We note in passing that $a$ is unitarily equivalent to the (unbounded) weighted shift operator on $\ell_{2}$ with weight sequence $\{n-1\}$.

Express $H=H^{2}(\mathbb{T}) \otimes \ell_{2}$. We take $b=a \otimes I$. (Countable) direct sums of closed operators are themselves closed, and since $b$ can be thought of as a direct sum of scalar multiples of $a$, we see that it too is closed. Take $c=I \otimes d$. Finally, we set $y=\sum \frac{1}{n} 1 \otimes f_{n}$ and write $\phi \in H^{*}$ for the linear functional $h \rightarrow\langle h, y\rangle$. 
Now let $\lambda \in \mathbb{C}$ and $\epsilon>0$ be given. Write $g$ for the exponential function $g(z)=$ $e^{\lambda z}$. We take $x_{n}=n g \otimes f_{n}$. Brief computations verify that each $x_{n}$ satisfies (1) and (2). For (3) it suffices to choose $n$ sufficiently large since $\|c x\|=\frac{\|g\|}{n}$.

Example 4.2. There is a linear subspace $S$ of $B\left(\mathbb{C}^{3}, H \oplus \mathbb{C} \oplus H\right)$ having the following properties:

(1) $S$ is closed in the strong operator topology.

(2) $S$ admits separating vectors $x$ and $y$ such that $S x \perp S y$.

(3) $S$ is not reflexive.

Construction. Take $H, b, c$, and $\phi$ as in the preceding example. For each $f \in$ Domain $b$, define $s_{f} \in B\left(\mathbb{C}^{3}, H \oplus \mathbb{C} \oplus H\right)$ by

$$
s_{f}\left[\begin{array}{l}
\lambda \\
\mu \\
\nu
\end{array}\right]=\left[\begin{array}{ccc}
f & b f & 0 \\
0 & \phi f & 0 \\
0 & 0 & c f
\end{array}\right]\left[\begin{array}{l}
\lambda \\
\mu \\
\nu
\end{array}\right]=\left[\begin{array}{c}
\lambda f+\mu b f \\
\mu \phi f \\
\nu c f
\end{array}\right], \quad \lambda, \mu, \nu \in \mathbb{C} .
$$

We take $S=\left\{s_{f} \in B\left(\mathbb{C}^{3}, H \oplus \mathbb{C} \oplus H\right): f \in\right.$ Domain $\left.b\right\}$.

The linearity of the map $f \rightarrow s_{f}$ makes it clear that $S$ is a linear space. Norm closure of $S$ follows from the fact that $b$ is a closed operator; thus we get (1) by finite-dimensionality of the domain $\mathbb{C}^{3}$. The vectors $x=\left[\begin{array}{l}1 \\ 0 \\ 0\end{array}\right]$ and $y=\left[\begin{array}{l}0 \\ 0 \\ 1\end{array}\right]$ satisfy the demands of (2).

We complete the proof by showing that $r \equiv\left[\begin{array}{lll}0 & 0 & 0 \\ 0 & 1 & 0 \\ 0 & 0 & 0\end{array}\right] \in \operatorname{ref} S$. Given a vector $x=\left[\begin{array}{l}\lambda \\ \mu \\ \nu\end{array}\right] \in \mathbb{C}^{3}$, we must be able to make $\left\|\left(s_{f}-r\right) x\right\|$ arbitrarily small for appropriate $f$. If $\mu=0$, we simply take $f=0$, so we may as well assume $\mu=1$. Thus we must simultaneously control $\|(b+\lambda I) f\|,|\phi f-1|$, and $\|\nu c f\|$, but that is precisely what Example 4.1 guarantees.

Proof of Theorem 1.6. Use Equation 3.2 on page 577 of [3] to embed Example 4.2 in a singly generated operator algebra.

Proof of Theorem 1.7. Write $a$ for the forward shift operator on $\ell_{2}$ with weight sequence $\left\{2^{-n}\right\}$ and take $A$ to be the strongly closed operator algebra generated by $a$. On page 724 of [6], A. Lambert observes that $A$ is strictly cyclic, but fails to be reflexive. Since $A$ is abelian, it follows that each non-zero vector in $\ell_{2}$ must separate $A$.

We mention in closing that Theorem 1.7 can also be established by applying the embedding of [3] to the following example.

Example 4.3. There is a linear subspace $S$ of $B\left(\mathbb{C}^{2}, H\right)$ having the following properties:

(1) $S$ is closed in the strong operator topology.

(2) Every non-zero vector separates $S$; some of these vectors are strictly separating.

(3) $S$ is not reflexive. 
Construction. Fix an injective quasi-nilpotent operator $a \in B(H)$. For each $f \in H$, define $s_{f}: \mathbb{C}^{2} \rightarrow H$ by

$$
s_{f}\left[\begin{array}{l}
\lambda \\
\mu
\end{array}\right]=\left[\begin{array}{ll}
f & a f
\end{array}\right]\left[\begin{array}{l}
\lambda \\
\mu
\end{array}\right]=\lambda f+\mu a f, \quad \lambda, \mu \in \mathbb{C} .
$$

Take $S \equiv\left\{s_{f}: f \in H\right\}$.

(1) and (2) are clear. For (3), fix a non-zero vector $g \in H$ and take $r \equiv\left[\begin{array}{ll}g & 0\end{array}\right]$. To complete the proof, it suffices to show that $r \in \operatorname{ref} S$. Given $x=\left[\begin{array}{l}\lambda \\ \mu\end{array}\right] \in \mathbb{C}^{2}$, we must be able to make $\left\|\left(s_{f}-r\right) x\right\|$ arbitrarily small for appropriate $f$. If $\mu=0$, we simply take $f=g$, so we may as well assume $\mu=1$. Thus we must control $\|(a+\lambda I) f-\lambda g\|$. In fact, we can always make this norm vanish: take $f=0$ if $\lambda=0$, and $f=\lambda(a+\lambda I)^{-1} g$ otherwise.

\section{REFERENCES}

1. E. A. Azoff, On finite rank operators and preannihilators, Memoirs Amer. Math. Soc. 357 (1986). MR 88a:47041

2. E. A. Azoff and H. A. Shehada, Algebras generated by mutually orthogonal idempotent operators, J. Operator Theory 29 (1993), 249-267. MR 95h:47063

3. E. A. Azoff and H. A. Shehada, Literal embeddings of linear spaces of operators, Indiana Univ. Math. J. 22 (1993), 571-589. MR 94i:47068

4. L. Ding, Separating vectors and reflexivity, Lin. Alg. Appl. 174 (1992), 37-52. MR 94a:47075

5. L. Ding, On strictly separating vectors and reflexivity, Operator Theory \& Integral Equations 19 (1994), 373-380. CMP 94:15

6. A. Lambert, Strictly cyclic operator algebras, Pac. J. Math. 39 (1971), 717-726. MR 46:9762

7. W.R. Wogen, Some counterexamples in nonselfadjoint algebras, Ann. of Math. 126 (1987), 415-427. MR 89b:47066

Department of Mathematics, University of Georgia, Athens, Georgia 30602-7403

E-mail address: azoff@alpha.math.uga.edu

Department of Mathematics and Computer Science, Georgia State University, AtLANTA, GEORGIA 30303-3083

E-mail address: matlfd@gsusgi2.gsu.edu

Department of Mathematics, University of North Carolina, Chapel Hill, North CarOLINA 27599

E-mail address: wrw@math.unc.edu 